Article

\title{
The Quest for Low Loss High Refractive Index Dielectric Materials for UV Photonic Applications
}

\author{
Yael Gutiérrez, Dolores Ortiz, José M. Saiz@ ${ }^{\circledR}$ Francisco González, Pablo Albella *(i) \\ and Fernando Moreno *(i) \\ Department of Applied Physics, University of Cantabria, Avda. Los Castros, s/n, 39005 Santander, Spain; \\ gvelay@unican.es (Y.G.); ortizd@unican.es (D.O.); saizvj@unican.es (J.M.S.); gonzaleff@unican.es (F.G.) \\ * Correspondence: pablo.albella@unican.es (P.A.); morenof@unican.es (F.M.)
}

Received: 26 September 2018; Accepted: 22 October 2018; Published: 25 October 2018

check for updates

\begin{abstract}
Nanostructured High Refractive Index (HRI) dielectric materials, when acting as nanoantennas or metasurfaces in the near-infrared (NIR) and visible (VIS) spectral ranges, can interact with light and show interesting scattering directionality properties. Also, HRI dielectric materials with low absorption in these spectral ranges show very low heat radiation when illuminated. Up to now, most of the studies of these kind of materials have been explored in the VIS-NIR. However, to the best of our knowledge, these properties have not been extended to the ultraviolet (UV), where their application in fields like photocatalysis, biosensing, surface-enhanced spectroscopies or light guiding and trapping can be of extraordinary relevance. Here, we present a detailed numerical study of the directional scattering properties, near-field enhancement and heat generation of several materials that can be good candidates for those applications in the UV. These materials include aluminum phosphide, aluminum arsenide, aluminum nitride, diamond, cerium dioxide and titanium dioxide. In this study, we compare their performance when forming either isolated nanoparticles or dimers to build either nanoantennas or unit cells for more complex metasurfaces.
\end{abstract}

Keywords: high refractive index dielectrics; UV plasmonics; scattering

\section{Introduction}

Plasmonics has been an extremely active area of research in the last decade [1]. The potential of using metallic nanoparticles (NPs) as electromagnetic field enhancers has opened up new possibilities in fields like chemistry, surface-enhanced spectroscopies, nanosensing, and medicine [2]. Although, in general, metallic NPs exhibit good plasmonic performance [3,4], their intrinsic Joule losses limit their use for many applications $[5,6]$. To overcome this problem, a lot of attention has been paid to dielectric nanostructures made of High-Refractive-Index (HRI) materials, working in the Visible (VIS) and Near-infrared (NIR) ranges. These materials are characterized by a high real part $n>2$ and a low imaginary part $k \approx 0$ of their refractive indices (some examples can be found in ref. [7]). They show the "ability" of enhancing the electromagnetic field both in the near and far field regimes without much absorption $[5,6,8,9]$ in those spectral ranges. In addition, they support magnetic resonances originated from the excited displacement currents. Moreover, HRI dielectric NPs manifest interesting properties of scattering directionality arising from the coherent effects between the excited electric and magnetic dipolar components [9-11]. Under some specific conditions, scattered radiation from a single HRI dielectric spherical nanoparticle can be concentrated either in the backward or forward scattering regions. When the electric and magnetic dipoles oscillate in- or out-of-phase, two special situations appear, the so-called "zero-backward condition" (null scattering in the backward direction) and the "minimum forward condition" (minimum scattering in the forward direction), respectively. Up to now, most of the studies concerning these effects have been done in the visible (VIS) and near-infrared (NIR) 
ranges using common semiconductors such as $\mathrm{Si}[12,13]$ or GaAs [14], because of their interest for optical communications applications.

Unlike metals, when HRI dielectric materials show negligible absorption in the VIS-NIR spectral ranges (see ref. [7] for some examples), no localized heat appears. Consequently, neither the surrounding medium nor the nanostructure is heated, something that could have a clear negative effect. For instance, it could limit the performance of nan-antennas $[15,16]$ by altering the refractive index of both the nanostructure and surrounding medium through the thermo-optic effect [17]. Also, depending on the incident power, the nanoantenna could be reshaped, or even melted [18]. In addition, the heat generated by the nanoparticle can either vaporize the solvent media, affect or even destroy molecules and proteins. Heat can also affect the SERS signal, and has connection to problems or anomalies found in several processes including thermal annealing, modified adsorption/desorption kinetics of surface molecules, and changes in the dielectric properties of both, medium and particle [19]. In consequence, HRI dielectric materials have been proposed as an alternative to metals in surface-enhanced spectroscopies $[5,6]$.

Extending plasmonics into the UV ( $3-6 \mathrm{eV}$ or $200<$ wavelength $<400 \mathrm{~nm}$ ) has triggered immense interest due to new challenges arising in specific areas [20]. For example, many biological compounds such as nucleotide bases, nucleic acids, or aromatic amino acids in cells have absorption bands in the ultraviolet (UV). By using the local electric field enhancement generated by nanoparticles when illuminated at resonant wavelength, surface enhanced spectroscopy techniques [21-23] (SERS, TERS, etc.) can be improved to ease the detection of biomolecules. Other biosensing techniques rely on monitoring the spectral shifts induced by some target analytes in the extinction peaks of the NPs acting as nanoantennas. Bioimaging can also benefit from using UV radiation, since the diffraction limit is proportional to the wavelength (the lower the wavelength the higher the resolution). Photocatalytic processes take also advantage of the UV radiation [24-26]. This area has experienced a great development in the last decade. In photocatalysis, photons are used to activate chemical reactions by offering product selectivity. This effect can be enhanced by plasmonic metals by using metal-semiconductor nanostructures, like $\mathrm{Au}-\mathrm{TiO}_{2}$ decorated particles $[27,28]$. However, more recently it was demonstrated that metallic NPs can drive chemical reactions on their own [29]. Most of these studies on UV plasmonics have been done using metals with strong UV plasmonic response such as aluminum, magnesium, gallium, or rhodium [20,30], but, to the best of our knowledge, HRI dielectric NPs have not been explored in this spectral range yet.

Here we present a numerical analysis of several candidate dielectric materials that show HRI dielectric properties at energies above $3 \mathrm{eV}$. The dielectric materials chosen for this study are aluminum phosphide (AlP), aluminum arsenide (AlAs), aluminum nitride (AlN), diamond, cerium dioxide $\left(\mathrm{CeO}_{2}\right)$, and titanium dioxide $\left(\mathrm{TiO}_{2}\right) . \mathrm{TiO}_{2}$ and $\mathrm{CeO}_{2}$ are commonly used in photocatalysis [31,32]. $\mathrm{AlN}, \mathrm{AlP}$, and AlAs have undergone extensive study because of their potential use in the fabrication of light-emitting diodes (LEDs) [33]. In fact, in 2010 an AlN LED was presented with an emission wavelength of $210 \mathrm{~nm}$, the shortest ever reported [34]. AlP and AlAs are materials commonly alloyed with $\mathrm{GaP}$ and $\mathrm{GaAs}$ to form $\mathrm{AlGaP}$ and $\mathrm{Al}_{x} \mathrm{G}_{1-\mathrm{x}} \mathrm{As}$ for their use in LED design. Nanodiamonds are being used in several areas of research and for various applications that include catalysis, drug delivery, and biomedical imaging [35].

The analysis of the aforementioned materials consists in a detailed numerical investigation of their directionality properties and their near-field enhancement in the UV. For the sake of comparison, we specifically consider isolated spheres of different sizes. It is worth mentioning that the results obtained for the spheres can be generalized for other NP's shapes. To mimic common typical experimental conditions, interaction effects in dimers are explored by comparing the near-field enhancement and the temperature generated in the nanoparticles and its surroundings.

This work is aimed to introduce some HRI dielectric materials that can offer high quality directional properties and high near-field enhancement without heat generation in the UV regime. These features are closely connected with UV light-trapping applications and sensing. We envision 
that this study can open a new landscape for optimizing the efficiency of solar energy harvesting, photocatalysis, photodetection, and spectroscopic techniques in the UV regime.

The work is organized as follows. In Section 2, we briefly review the theoretical concepts and methods used to perform the electromagnetic and temperature calculations. In Section 3, we describe the criteria used to select the analyzed materials. In addition, the optical properties of these materials are presented. Section 4 includes the results and discussion of this research: an analysis of the near-field enhancement, directionality properties and cooperative effects in spherical NPs made of the selected materials. Finally, in Section 5 the main conclusions of this work are summarized.

\section{Methods}

\subsection{Electromagnetic Simulations}

The electromagnetic interaction between isolated nanospheres and light has been modeled following the Lorenz-Mie formalism for scattering and absorption of light by small particles [36,37]. The scattering efficiency by a spherical particle is given by

$$
Q_{s c a}=\frac{2}{x^{2}} \sum_{n=1}^{\infty}(2 n+1)\left(\left|a_{n}\right|^{2}+\left|b_{n}\right|^{2}\right),
$$

where $a_{n}$ and $b_{n}$ are the scattering coefficients of the Lorenz-Mie theory. These depend on both particle size and optical properties of the NP relative to its surrounding medium. The size parameter $x$ is given by $2 \pi m_{\text {med }} R / \lambda$, where $\lambda$ is the wavelength of incident light in the surrounding medium of refractive index $m_{\text {med }}$, and $R$ is the radius of the sphere. From a physical point of view, coefficients $a_{n}$ and $b_{n}$ represent the weighting factors of the different multipolar contributions of order $n$, electric or magnetic, respectively. In particular, $a_{1}$ and $b_{1}$ correspond to the electric and magnetic dipolar modes, respectively. When $a_{1}=b_{1}$ the zero backward condition is fulfilled and, as a result [9], there is null scattering in the backward direction, assuming that the rest of the coefficients are negligible (small size limit).

In the far-field region, the scattered electric field $E^{s c a}$ is related to the incident one $E^{i n c}$ through the scattering amplitude matrix $S(\theta, \phi)[36]$. The scattered $p$-/s-components are given by

$$
\left(\begin{array}{c}
E_{p}^{s c a} \\
E_{s}^{s c a}
\end{array}\right) \propto\left(\begin{array}{ll}
S_{2}(\theta, \phi) & S_{3}(\theta, \phi) \\
S_{4}(\theta, \phi) & S_{1}(\theta, \phi)
\end{array}\right)\left(\begin{array}{c}
E_{p}^{i n c} \\
E_{s}^{i n c}
\end{array}\right),
$$

where $\theta$ and $\phi$ are the scattering and azimuthal angles, respectively. The $S_{i}(\theta, \phi)$ elements are related to the coefficients $a_{n}$ and $b_{n}$, and specific angular functions [36].

In order to quantify the efficiency of the zero backward scattering condition, the forward to backward scattering intensity ratio $\left(I_{F w d} / I_{B c k}\right)$ is introduced. This magnitude is defined as the ratio between the integrated scattered intensity in the forward and backward hemispheres. A key point to consider is that although a particle can have a high value of $I_{F w d} / I_{B c k}$, its scattering efficiency may be negligible, making its scattering directionality properties useless. Therefore, it is important to find NPs made of materials that have high values of both $I_{F w d} / I_{B c k}$ and $Q_{s c a}$ [38]. Keeping this in mind, we have evaluated the product between these two magnitudes: $Q_{Z B}=I_{F w d} / I_{B c k} \cdot Q_{s c a}$. We will refer to this magnitude as zero backward efficiency $\left(Q_{Z B}\right)$.

As aforementioned, the electromagnetic response of the nanospheres excited with light has been modeled using the Lorenz-Mie formalism. On the other hand, the interaction of light with dimers has been solved with finite element (FEM) calculations through commercial software COMSOL Multiphysics 5.2 (COMSOL Inc., Burlington, MA, USA) [39]. 


\subsection{Temperature Calculations}

The general heat generation process involves optical absorption, heat generation by itself and heat transfer to the surrounding medium. For the mechanism of heat release, the electric field strongly drives mobile carriers inside the NPs, and the energy gained turns into heat. Then, the heat diffuses away from the NP and leads to an increase of the temperature of the surrounding medium [40]. In metallic nanoparticles, the heat generation rate is especially strong under plasmon resonance. However, in semiconductors, this is much weaker since heat dissipation occurs through an interband absorption process with the creation of a single mobile electron-hole pair (exciton). In absence of phase transformations, the temperature distribution around an optically excited NP is described by the heat transfer equation $[40,41]$

$$
\rho(\boldsymbol{r}) c(\boldsymbol{r}) \frac{\partial T(\boldsymbol{r}, t)}{\partial t}=\nabla k(\boldsymbol{r}) \nabla T(\boldsymbol{r})+Q_{e}(\boldsymbol{r}, t),
$$

where $r$ and $t$ are the spatial coordinate and time, respectively. $T(r, t)$ is the local temperature, and the material parameters $\rho(r), \mathrm{c}(r)$ and $\kappa(r)$ are the density, specific heat and thermal conductivity, respectively. The magnitude $Q_{e}(r, t)$ represents a heat source produced by light dissipation in NPs. This term is composed by both resistive and magnetic losses. $Q_{e}(r, t)$ has been obtained by solving a system of Maxwell's equations including appropriate boundary conditions [5]. In our case, the whole process of light absorption and subsequent heat transfer between the nanostructure and the surrounding medium has been modeled by means of finite element simulations implemented in COMSOL Multiphysics 5.2 [39] (following the same method as in [2,3]).

\section{Materials}

In the search for materials with an appropriate HRI dielectric properties in the UV range, we look for real materials whose optical properties fulfill the two following constrains on their dielectric constant $\varepsilon=\varepsilon_{1}+\mathrm{i} \varepsilon_{2}$,

- A real part of the dielectric permittivity $\varepsilon_{1}>4$ in the UV allow the generation of electric and magnetic dipolar responses in the nanoparticle. This is necessary to satisfy the zero backward and minimum forward conditions.

- An imaginary part of the dielectric permittivity that meets the condition $\varepsilon_{2}<1$ at energies higher than $3 \mathrm{eV}$. This opens a low-absorption window above $3 \mathrm{eV}$, since it can be easily derived that, provided that $\varepsilon_{1}>\varepsilon_{2}$, the corresponding imaginary part of the refractive index $\left(n_{i} \approx \varepsilon_{2} / 2 \sqrt{\varepsilon_{1}}\right)$ would be of the order of 0.1 .

The materials we have analyzed that fulfill these requirements are the following: aluminum phosphide (AlP), aluminum arsenide (AlAs), aluminum nitride (AlN), diamond, cerium dioxide $\left(\mathrm{CeO}_{2}\right)$, and titanium dioxide $\left(\mathrm{TiO}_{2}\right)$. Table 1 shows the photon energy at which $\varepsilon_{2}=1$ for each of the selected materials. In the case of AlN and diamond, the photon energy at which this condition is reached is out of the spectral range considered in this study $(1-6 \mathrm{eV})$.

Table 1. Photon energy at which $\varepsilon_{2}=1$ for each of the selected materials. For AlN and diamond, the dash indicates that the $\varepsilon_{2}=1$ is produced outside of the spectral range under study $(1-6 \mathrm{eV})$.

\begin{tabular}{cc}
\hline Material & $\boldsymbol{E}\left(\varepsilon_{\mathbf{2}}=\mathbf{1}\right) / \mathbf{e V}$ \\
\hline $\mathrm{AlP}$ & 3.90 \\
$\mathrm{AlAs}$ & 3.25 \\
$\mathrm{AlN}$ & - \\
Diamond & - \\
$\mathrm{CeO}_{2}$ & 3.53 \\
$\mathrm{TiO}_{2}$ & 3.39 \\
\hline
\end{tabular}


Some of the wide-bandgap materials have been rejected because they do not fulfill one of the two constraints. This includes GaN, $\mathrm{ZnS}, \mathrm{ZnO}$, and AlSb. For all the selected materials, with the exception of AlP and AlAs, NP fabrication methods have been reported and are available in the literature $[35,42-44]$.

Figure 1a-f shows the dielectric permittivity $\left(\varepsilon=\varepsilon_{1}+i \varepsilon_{2}\right)$ of the selected materials. The region where $\varepsilon_{1}>4$ and $\varepsilon_{2}<1$ within the UV is shadowed in gray. In Figure $1 \mathrm{~g}$, h, the dielectric function of Rh and $\mathrm{Al}$ is shown as a reference because these metals are widely used in UV plasmonic applications [20]. Figure $1 \mathrm{i}$ plots the dielectric permittivity of the HRI dielectric materials taken as reference, like $\mathrm{Si}$, $\mathrm{GaAs}$ and $\mathrm{Ge}$. In the case of the reference materials ( $\mathrm{Al}, \mathrm{Rh}, \mathrm{Si}, \mathrm{GaAs}$, and $\mathrm{Ge}$ ) the shadowed region indicates the UV spectral range.

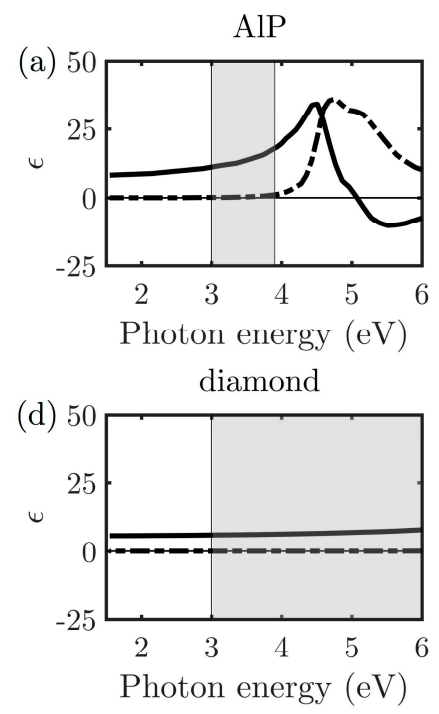

$\mathrm{Al}$

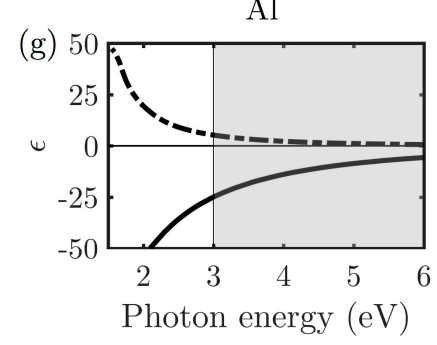

AlAs
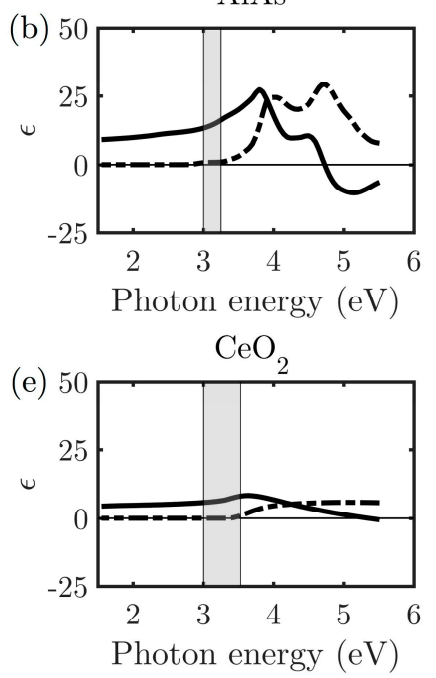

$\mathrm{Rh}$

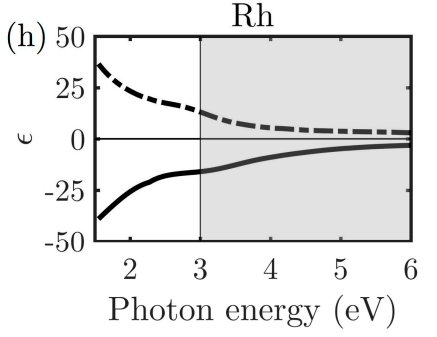

AlN
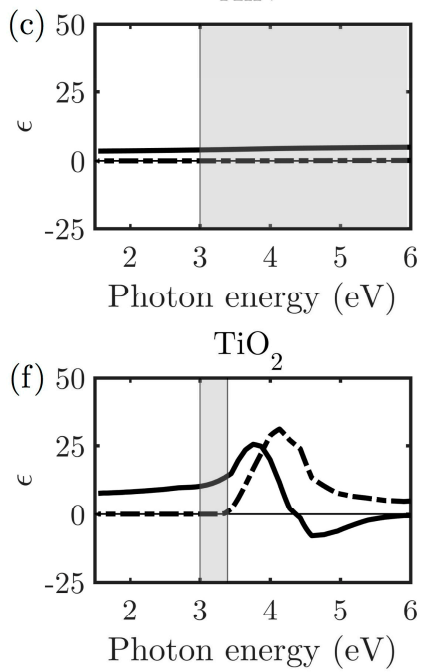

Reference dielectrics

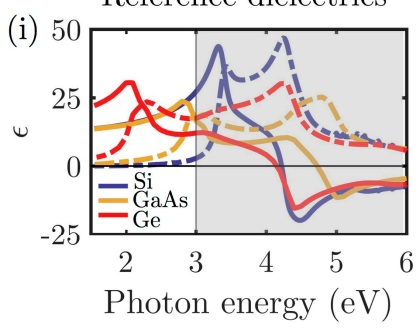

Figure 1. Real (solid line) and imaginary (dashed line) parts of the dielectric permittivity $\left(\varepsilon=\varepsilon_{1}+i \varepsilon_{2}\right)$ of (a) AlP [45], (b) AlAs [46], (c) AlN [47], (d) diamond, (e) $\mathrm{CeO}_{2}$ [48], (f) $\mathrm{TiO}_{2}$ [49], (g) $\mathrm{Al}$, (h) $\mathrm{Rh}$ [50] and (i) dielectrics taken as reference like $\mathrm{Si}$ (blue), GaAs (yellow) and Ge (red) [50]. The region in which $\varepsilon_{2}<1$ and $\varepsilon_{1}>4$ within the UV is shadowed in gray. In the case of the reference materials ( $\mathrm{Al}, \mathrm{Rh}, \mathrm{Si}$, GaAs and $\mathrm{Ge}$ ) the shadowed region indicates the UV spectral range.

\section{Results}

\subsection{Near-Field Enhancement}

Near-field enhancement produced by NPs when electromagnetically irradiated is essential for applications like SERS (surface enhanced Raman spectroscopy) or SEF (surface enhanced fluorescence). In these spectroscopy techniques, most of the NP surface is covered with the molecules under study, so the electromagnetic field acting on them can be approximated by an average of the electric field produced over the whole NP surface [6,21,51]. Figure 2 shows the near-field enhancement averaged over the NP surface $\left\langle|E| /\left|E_{0}\right|\right\rangle$. Spheres with $R$ varying from 30 to $90 \mathrm{~nm}$ have been considered. With dashed and solid white lines are highlighted the energy at which $\varepsilon_{2}=1$ and the starting energy of the UV regime respectively $(3 \mathrm{eV})$. For reference, $\left\langle|E| /\left|E_{0}\right|\right\rangle$ for two common metals for UV 
plasmonic applications are included: $\mathrm{Rh}$ and $\mathrm{Al}$. In the case of $\mathrm{Al}$, a realistic $5 \mathrm{~nm}$ thick oxide $\left(\mathrm{Al}_{2} \mathrm{O}_{3}\right)$ shell has been considered [52,53].

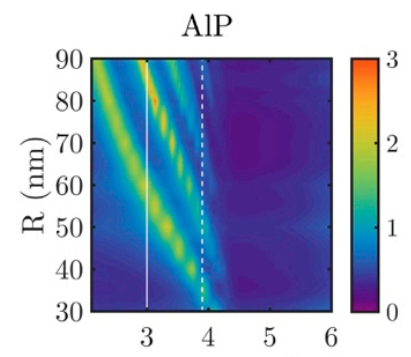

Photon energy $(\mathrm{eV})$

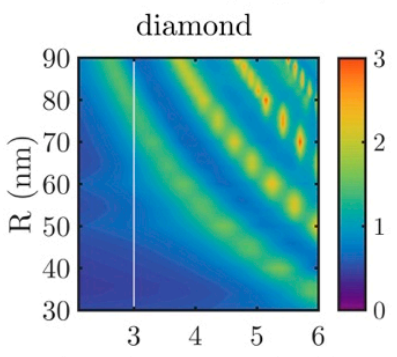

Photon energy $(\mathrm{eV})$

$\mathrm{Si}$

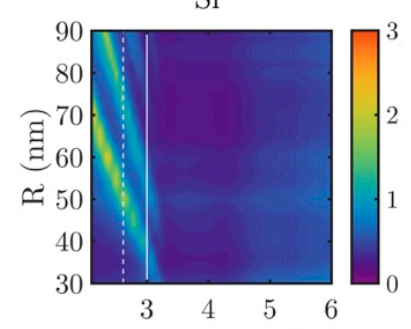

Photon energy $(\mathrm{eV})$

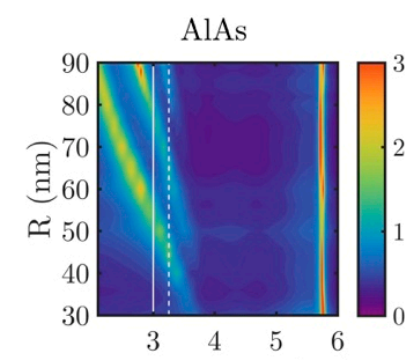

Photon energy $(\mathrm{eV})$

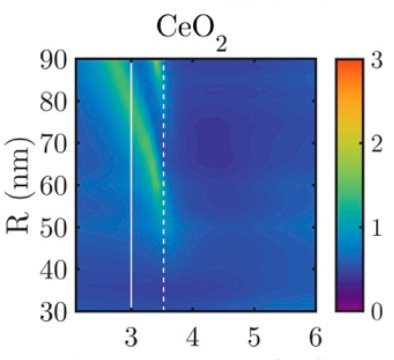

Photon energy $(\mathrm{eV})$

$\mathrm{Al}$

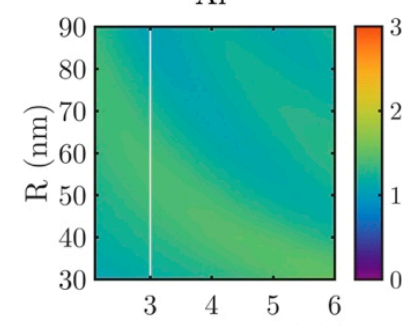

Photon energy $(\mathrm{eV})$

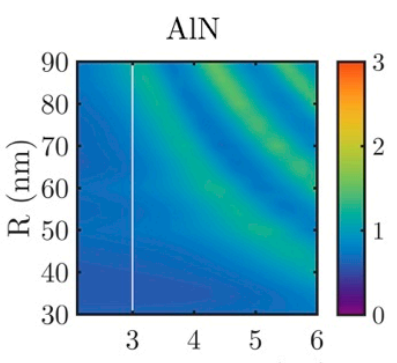

Photon energy (eV)

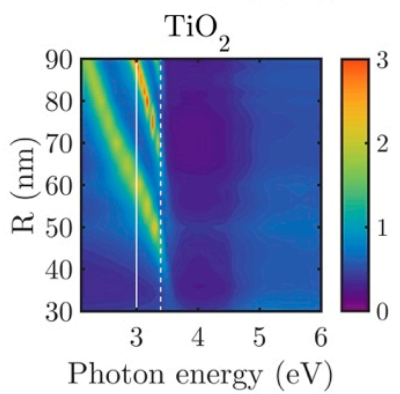

$\mathrm{Rh}$

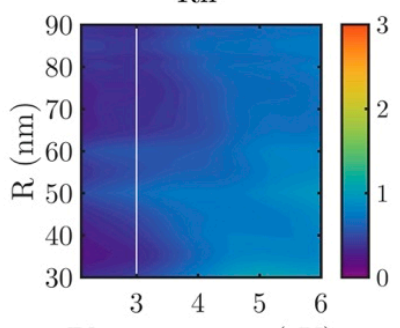

Photon energy (eV)

Figure 2. Near-field enhancement averaged over the surface $\left\langle|E| /\left|E_{0}\right|\right\rangle$ of spheres with radius $R$ (between 30 and $90 \mathrm{~nm}$ ). A white dashed line represents the energy where $\varepsilon_{2}=1$, and a white solid line indicates the energy where we consider the UV regime starts, $3 \mathrm{eV}$.

Unlike $\mathrm{Si}$, all the HRI dielectric materials analyzed in this research, exhibit its electric field enhancement at energies above $3 \mathrm{eV}$. AlP, $\mathrm{AlAs}, \mathrm{CeO}_{2}$ and $\mathrm{TiO}_{2}$ also show similar performance and high values of $\left\langle|E| /\left|E_{0}\right|\right\rangle$ can be reached between 3 and 3.4 or $4 \mathrm{eV}$ depending on the material. We would like to emphasize that $\mathrm{TiO}_{2}$ shows the largest enhancement at energies above $3 \mathrm{eV}$. AlN and diamond also have very similar behavior since their absorption bands are above $6 \mathrm{eV}$ : they have a HRI dielectric like behavior all along the studied spectral range. In this case, diamond presents the highest enhancement.

The values of $\left\langle|E| /\left|E_{0}\right|\right\rangle$ for $\mathrm{Al}$ (with its native oxide shell included) and Rh NPs are also shown for comparison. Rhodium presents very low enhancement because its LSPRs are into deep UV (above $6 \mathrm{eV})[20,30]$. On the contrary, $\mathrm{Al} / \mathrm{Al}_{2} \mathrm{O}_{3}$ nanospheres present wider peaks with moderate intensity. Consequently, the value of $\left\langle|E| /\left|E_{0}\right|\right\rangle$ is almost constant for all sizes in the studied spectral range.

\subsection{Directionality Properties: The Full-Forward Condition}

The full-forward condition, also known as the first Kerker condition, maximizes the scattered intensity in the forward direction while it minimizes the backward one. This situation has found a great utility in light guiding and light trapping applications [11,54-57].

In order to evaluate the full-forward condition, we have considered nanospheres of different radii made of AlP, AlAs, $\mathrm{AlN}, \mathrm{CeO}_{2}, \mathrm{TiO}_{2}$ and $\mathrm{Si}$ and we have analyzed the following parameters: photon 
energy where the zero-backward condition is produced $\left(E_{Z B}\right)$, the scattering efficiency $\left(Q_{s c a}\right)$ and the forward to backward scattering ratios $\left(I_{F w d} / I_{B c k}\right)$ at $E_{Z B}$. In addition, the combined effect of these two last magnitudes, the zero backward efficiency, defined as $Q_{Z B}=Q_{s c a} \cdot I_{F w d} / I_{B c k}$ will be also analyzed.

Figure 3a shows the photon energies where the zero-backward condition holds $\left(E_{Z B}\right)$ for spheres of radius $R$ between 30 and $90 \mathrm{~nm}$. The zero-forward condition is not satisfied for all the materials in the analyzed size range. For example, for $\mathrm{CeO}_{2} \mathrm{NPs}, R$ should be larger than $60 \mathrm{~nm}$. On the contrary, nanospheres made of either AlP or diamond, exhibit this condition for all analyzed radii. The rest of materials also shows the zero-backward condition in the UV but not for all radii. Notice that all of them satisfy this condition at higher energies than Si. Those that show the zero-backward condition above $3 \mathrm{eV}$ are AlP $(30 \leq R \leq 50 \mathrm{~nm})$, $\mathrm{AlN}(40 \leq R \leq 80 \mathrm{~nm})$, diamond $(40 \leq R \leq 70 \mathrm{~nm}), \mathrm{CeO}_{2}$ $(60 \leq R \leq 80 \mathrm{~nm})$, and $\mathrm{TiO}_{2}(R=50 \mathrm{~nm})$.
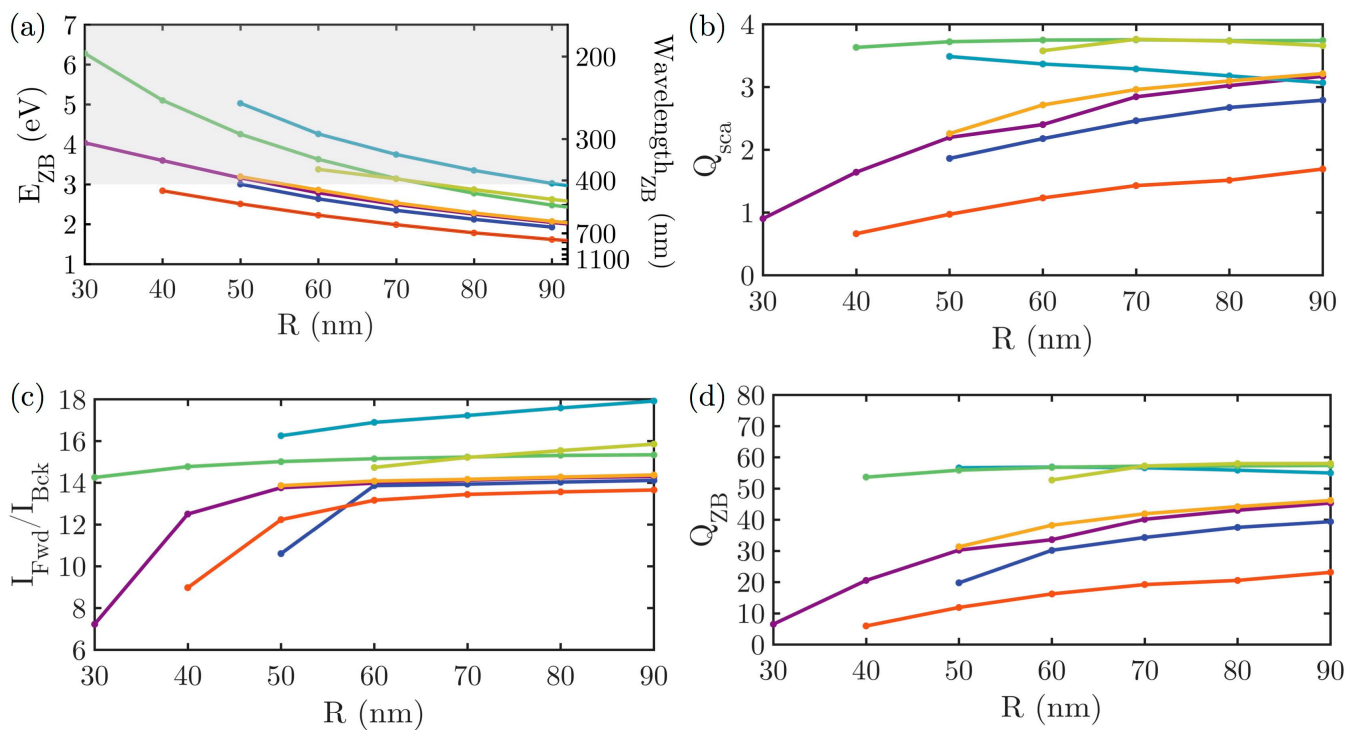

$$
\rightarrow \mathrm{AlP} \rightarrow \mathrm{AlAs} \rightarrow \mathrm{AlN} \rightarrow \text { diamond } \rightarrow \mathrm{CeO}_{2} \rightarrow \mathrm{TiO}_{2} \rightarrow \mathrm{Si}
$$

Figure 3. (a) Photon energies where the zero-backward condition holds $\left(E_{Z B}\right)$, (b) scattering efficiency $Q_{s c a}$ at the photon energy of the zero-backward condition, (c) forward to backward intensity ratio $I_{F w d} / I_{B c k}$ at the zero-backward condition and (d) zero backward efficiency, $Q_{Z B}$, for spheres of radius $R$ (between 30 and $90 \mathrm{~nm}$ ) made of different materials.

High values of $Q_{s c a}$ are desirable in order to exploit the maximum amount of incoming intensity in the analyzed condition, the zero-backward [38]. Figure $3 b$ shows the scattering efficiency $Q_{s c a}$ at the photon energy of the zero-backward condition as a function of the sphere radius, $R$. Among the studied materials, those that show the highest values of $Q_{s c a}$ at the zero-backward condition are diamond, $\mathrm{CeO}_{2}$ and $\mathrm{AlN}$. AlP, one of the promising candidates for directionality applications in the UV, presents values of $Q_{s c a}$ around two times smaller than diamond and $\mathrm{CeO}_{2}$, and very similar to those of $\mathrm{TiO}_{2}$. It is worth noting that all the analyzed materials show higher values of $Q_{s c a}$ than $\mathrm{Si}$.

Figure $3 c$ shows the forward to backward intensity ratio $I_{F w d} / I_{B c k}$ at the photon energy of the zero-backward condition as a function of $R$. This magnitude will allow us to quantify the asymmetry introduced in the scattered intensity along the propagating direction when imposing the zero-backward conditions. Diamond, $\mathrm{CeO}_{2}$ and $\mathrm{AlN}$ are the materials that present higher ratios $I_{F w d} / I_{B c k}$. Values remain almost constant for all the considered sizes. $\mathrm{TiO}_{2}$ and AlP present the same values of $I_{F w d} / I_{B c k}$, and very similar to that of AlAs. Once again, Si shows the lowest ratio.

The ideal zero backward condition is a combination of two effects: a high scattering efficiency and a high forward to backward ratio. Therefore, forward directionality requires the presence of both. This makes it necessary to evaluate the zero backward efficiency, defined as $Q_{Z B}=Q_{s c a} \cdot I_{F w d} / I_{B c k}$. 
Figure $3 \mathrm{~d}$ shows the values of $Q_{Z B}$ at the photon energy of the zero-backward condition as a function of $R$. Diamond, $\mathrm{CeO}_{2}$ and AlN present the same constant value of $Q_{Z B}$, approximately. The zero backward efficiency for AlP and $\mathrm{TiO}_{2}$ takes the same values. However, in this case, $Q_{Z B}$ becomes smaller as $R$ decreases. Once again, $\mathrm{Si}$ is the material that shows the worst performance. Therefore, all the analyzed materials are better options than Si for scattering directionality applications, in both the VIS and UV ranges.

\subsection{Interaction Effects}

In this section, possible interaction effects are considered by studying the near-field enhancement that can be produced between two NPs in close interaction and the consequences that this interaction may have in the heat generation process. Interaction effects are widely used when designing nanostructures for surface enhanced spectroscopic techniques [6]. A dimer made of spheres with $R=70 \mathrm{~nm}$ and interparticle distances (gap) of 10 and $20 \mathrm{~nm}$ are analyzed.

$\mathrm{TiO}_{2}$ and diamond are selected as representative materials of the two types of HRI dielectrics studied previously: those with losses $\left(\varepsilon_{2}>0\right)$ above $3.5 \mathrm{eV}\left(\mathrm{AlP}, \mathrm{AlAs}, \mathrm{CeO}_{2}\right.$ and $\mathrm{TiO}_{2}$ ) and those with losses above $6 \mathrm{eV}$ (AlN and diamond). The selected materials for this study $\left(\mathrm{TiO}_{2}\right.$ and diamond) when studied as isolated spheres, showed the highest value of $\left\langle|\mathrm{E}| /\left|\mathrm{E}_{0}\right|\right\rangle$. We also present the behavior of $\mathrm{Al} \mathrm{NPs}$ as a reference. Once again, a $5 \mathrm{~nm}$ oxide shell $\left(\mathrm{Al}_{2} \mathrm{O}_{3}\right)$ has been considered in order to mimic common experimental situations [52].

Figure 4 shows the spectral values of $|E| /\left|E_{0}\right|$ and $\Delta T$ (temperature increase with respect to ambient temperature) at the center of the gap of dimers made of $\mathrm{Al}+\mathrm{Al}_{2} \mathrm{O}_{3}, \mathrm{TiO}_{2}$ and diamond. In all cases, the dimers are excited with a plane wave linearly polarized along the dimer axis (see inset in Figure 4a). This polarization is chosen to enhance the interaction effects [58]. By comparing the values of $|E| /\left|E_{0}\right|$ for the different materials analyzed, both diamond and $\mathrm{TiO}_{2}$ produce the highest values above $3 \mathrm{eV}$. In the case of the $\mathrm{Al}+\mathrm{Al}_{2} \mathrm{O}_{3}$ dimer, the enhancement is significantly smaller than that of either diamond or $\mathrm{TiO}_{2}$ (around 4 times and one order of magnitude, respectively) and at an energy slightly below $3 \mathrm{eV}$. Also note that, for diamond and $\mathrm{TiO}_{2}$, the temperature does not increase $(\Delta T=0)$ when excited at the photon energies of maximum enhancement. However, for $\mathrm{Al} / \mathrm{Al}_{2} \mathrm{O}_{3}$, the temperature is increased
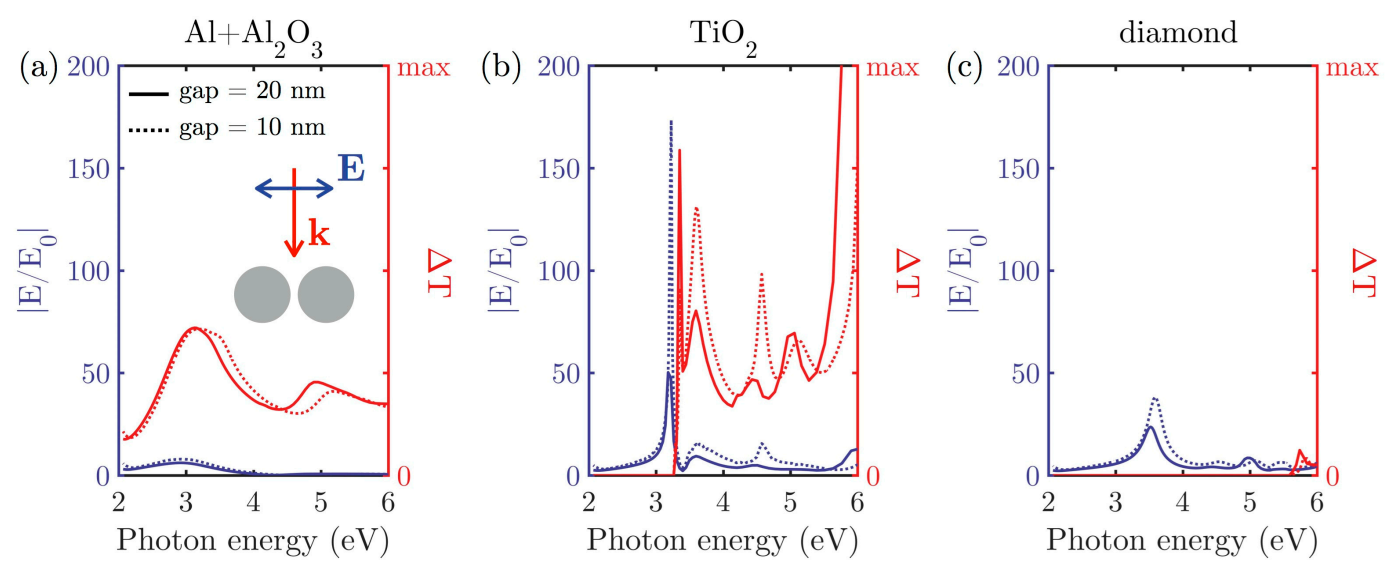

Figure 4. Electric field enhancement $|E| /\left|E_{0}\right|$ (blue line, left axis) and temperature difference with respect to ambient temperature $\Delta T$ (red line, right axis) at the center of the gap of (a) $\mathrm{Al}+\mathrm{Al}_{2} \mathrm{O}_{3}$, (b) $\mathrm{TiO}_{2}$ and (c) diamond dimers. The analyzed dimer configuration is shown as inset in (a): a dimer of spheres of $R=70 \mathrm{~nm}$ with gap $=10$ (dashed line) and $20 \mathrm{~nm}$ (solid line), illuminated with a plane wave linearly polarized along the dimer axis.

Similar results to those of $\mathrm{TiO}_{2}$ are expected for AlP, AlAs and $\mathrm{CeO}_{2}$ : high values of $|E| /\left|E_{0}\right|$ and low values of $\Delta T$ at energies below their corresponding absorption bands $(\approx 3.5 \mathrm{eV})$. At higher 
energies, $\Delta T$ increases while $|E| /\left|E_{0}\right|$ decreases. Also, mention that for AlN, a behavior similar to diamond is expected, moderate values of $|E| /\left|E_{0}\right|$ with $\Delta T=0$ for energies below $6 \mathrm{eV}$.

The main difference between having a gap of 10 or $20 \mathrm{~nm}$ is that for the shortest one both $|E| /\left|E_{0}\right|$ and $\Delta T$, are higher. This is caused by the strength of the interaction: as the distance between particles become smaller the interaction becomes stronger. Also, a red-shift of both $|E| /\left|E_{0}\right|$ and $\Delta T$ spectra is produced as the gap becomes shorter [58].

Figure 5 shows the near-field enhancement $\left(|E| /\left|E_{0}\right|\right)$ and heat maps $(\Delta T)$ for diamond and $\mathrm{Al}$ $+\mathrm{Al}_{2} \mathrm{O}_{3}$ dimers with a gap $=20 \mathrm{~nm}$. Since the temperature increment is proportional to the intensity of the illuminating beam, the temperature scale is arbitrary and for comparison purposes, going from 0 to a maximum value that depends on this intensity [40]. The diamond dimer offers the highest near field enhancement, together with the smallest heat perturbation. However, this is not the case for $\mathrm{Al}+\mathrm{Al}_{2} \mathrm{O}_{3}$, where the field enhancement is four times smaller and significant heat generation is present, as expected from its absorption [53]. Although the results for gap $=10 \mathrm{~nm}$ are not shown, the overall effect of decreasing the gap is an increase of the near-field enhancement and temperature at the center of the gap, while keeping a very similar $|E| /\left|E_{0}\right|$ and $\Delta T$ spatial distribution [2].

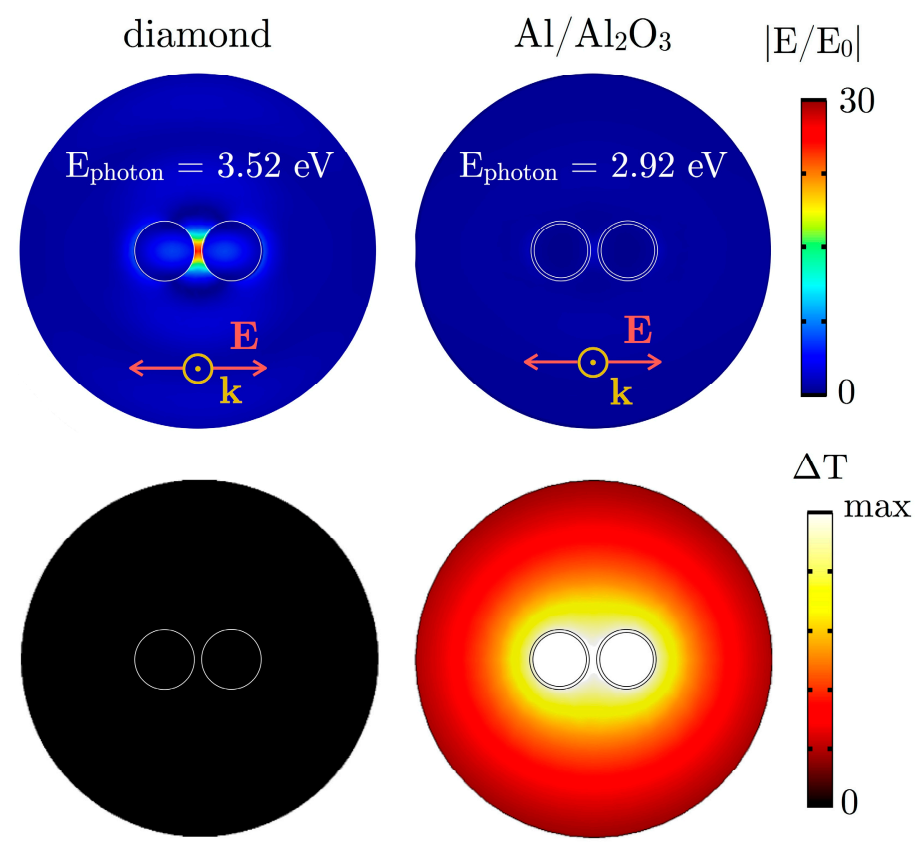

Figure 5. Near field |E / | E0| (top row) and temperature difference $\Delta \mathrm{T}$ (bottom row) maps for a diamond and $\mathrm{Al} / \mathrm{Al} 2 \mathrm{O} 3$ dimers with gap $=20 \mathrm{~nm}$. Both dimers are illuminated at resonance (photon energy indicated as a inset), with a p-polarized beam $\mathrm{E}$ and wave vector $\mathrm{k}$. E $E_{\text {photon }}$ corresponds to the photon energy of the illuminating beam. The chosen values of $E_{\text {photon }}$ are those that produce the maximum near-field enhancement at the center of the gap.

\section{Conclusions}

We have explored the possibilities of a selection of semiconductors-of the low loss High Refractive Index kind-to form nanoparticles with interesting applications in the UV region. The behavior we have considered of interest includes acting as HRI nanoantennas or behaving as unit cells of more complex metamaterials. The analyzed materials include some important semiconductors like AlP, AlAs, AlN, diamond, $\mathrm{CeO}_{2}$ and $\mathrm{TiO}_{2}$. When studying its near field-averaged over the surface of the nanoparticle-some important values of the enhancement have been observed within the UV range in most of the considered materials. In the far field, the possibility of using nanoparticles of these materials to generate light directionality effects has been explored by means of the zero-backward efficiency, which accounts for both the scattering efficiency and the backward-to-forward scattering 
ratio. In fact, all the analyzed materials are better options than $\mathrm{Si}$ for scattering directionality applications, both in the VIS and UV ranges. Finally, the near-field enhancement and local temperature effects have been studied for these materials when forming dimers, reaching high values of the near field, similar to the response of well-known semiconductors used in VIS and near-IR ( $\mathrm{Si}$, GaAs, and Ge).

Based on these analyses, some of the studied compounds can be considered as promising High Refractive Index dielectrics in the UV. On the one hand there is diamond, along with AlN, that show loss-less behavior below $6 \mathrm{eV}$, so they have HRI dielectric character in the entire optical regime (from near-IR to UV). They exhibit the highest values of $Q_{Z B}$, only matched by $\mathrm{CeO}_{2}$, but with moderate values of $|E| /\left|E_{0}\right|$ all along the UV range. On the other hand, $\mathrm{TiO}_{2}$, along with $\mathrm{AlP}, \mathrm{AlAs}$ and $\mathrm{CeO}_{2}$, present losses above $\approx 3.5 \mathrm{eV}$. Between 3 and $3.5 \mathrm{eV}$ they present high values of $|E| /\left|E_{0}\right|$ but lower $Q_{Z B}$ values than $\mathrm{AlN}$ and diamond.

Finally, the analysis of the thermal response in the case of dimers shows that, for both $\mathrm{TiO}_{2}$ and diamond, high values of $|E| /\left|E_{0}\right|$ can be generated at energies above $3 \mathrm{eV}$ without any losses and consequently no heat.

Author Contributions: Y.G. and F.M. conceived the original idea. Y.G. carried out the numerical calculations and wrote the paper. All authors analyzed the results and reviewed the manuscript. P.A. and F.M. supervised the study.

Funding: This research has been supported by the Army Research Laboratory under Cooperative Agreement Number W911NF-17-2-0023 and by SODERCAN (Sociedad para el Desarrollo de Cantabria) through the Research Vicerrectorate of the University of Cantabria.

Acknowledgments: Y.G. wants to thank the University of Cantabria for her FPU grant. P.A also acknowledges funding from the Ramon y Cajal Fellowship RYC-2016-20831.

Conflicts of Interest: The authors declare no conflict of interest.

\section{References}

1. Schuller, J.A.; Barnard, E.S.; Cai, W.; Jun, Y.C.; White, J.S.; Brongersma, M.L. Plasmonics for Extreme Light Concentration and Manipulation. Nat. Mater. 2010, 9, 193-204. [CrossRef] [PubMed]

2. Prasad, P.N. Nanophotonics; John Wiley \& Sons, Inc.: Hoboken, NJ, USA, 2004.

3. Blaber, M.G.; Arnold, M.D.; Ford, M.J. A Review of the Optical Properties of Alloys and Intermetallics for Plasmonics. J. Phys. Condens. Matter 2010, 22, 143201. [CrossRef] [PubMed]

4. Lalisse, A.; Tessier, G.; Plain, J.; Baffou, G. Plasmonic Efficiencies of Nanoparticles Made of Metal Nitrides (TiN, ZrN) Compared with Gold. Sci. Rep. 2016, 6, 38647. [CrossRef] [PubMed]

5. Albella, P.; Alcaraz de la Osa, R.; Moreno, F.; Maier, S.A. Electric and Magnetic Field Enhancement with Ultralow Heat Radiation Dielectric Nanoantennas: Considerations for Surface-Enhanced Spectroscopies. ACS Photonics 2014, 1, 524-529. [CrossRef]

6. Caldarola, M.; Albella, P.; Cortés, E.; Rahmani, M.; Roschuk, T.; Grinblat, G.; Oulton, R.F.; Bragas, A.V.; Maier, S.A. Non-Plasmonic Nanoantennas for Surface Enhanced Spectroscopies with Ultra-Low Heat Conversion. Nat. Commun. 2015, 6, 7915. [CrossRef] [PubMed]

7. Forouhi, A.R.; Bloomer, I. Optical Properties of Crystalline Semiconductors and Dielectrics. Phys. Rev. B 1988, 38, 1865-1874. [CrossRef]

8. Decker, M.; Staude, I. Resonant Dielectric Nanostructures: A Low-Loss Platform for Functional Nanophotonics. J. Opt. (UK) 2016, 18, 1-31. [CrossRef]

9. García-Etxarri, A.; Gómez-Medina, R.; Froufe-Pérez, L.S.; López, C.; Chantada, L.; Scheffold, F.; Aizpurua, J.; Nieto-Vesperinas, M.; Sáenz, J.J. Strong Magnetic Response of Submicron Silicon Particles in the Infrared. Opt. Express 2011, 19, 4815-4826. [CrossRef] [PubMed]

10. Geffrin, J.M.; García-Cámara, B.; Gómez-Medina, R.; Albella, P.; Froufe-Pérez, L.S.; Eyraud, C.; Litman, A.; Vaillon, R.; González, F.; Nieto-Vesperinas, M.; et al. Magnetic and Electric Coherence in Forward- and Back-Scattered Electromagnetic Waves by a Single Dielectric Subwavelength Sphere. Nat. Commun. 2012, 3, 1171. [CrossRef] [PubMed] 
11. Staude, I.; Miroshnichenko, A.E.; Decker, M.; Fofang, N.T.; Liu, S.; Gonzales, E.; Dominguez, J.; Luk, T.S.; Neshev, D.N.; Brener, I.; et al. Tailoring Directional Scattering through Magnetic and Electric Resonances in Subwavelength Silicon Nanodisks. ACS Nano 2013, 7, 7824-7832. [CrossRef] [PubMed]

12. Evlyukhin, A.B.; Reinhardt, C.; Seidel, A.; Luk'yanchuk, B.S.; Chichkov, B.N. Optical Response Features of Si-Nanoparticle Arrays. Phys. Rev. B 2010, 82, 045404. [CrossRef]

13. Zywietz, U.; Schmidt, M.K.; Evlyukhin, A.B.; Reinhardt, C.; Aizpurua, J.; Chichkov, B.N. Electromagnetic Resonances of Silicon Nanoparticle Dimers in the Visible. ACS Photonics 2015, 2, 913-920. [CrossRef]

14. Person, S.; Jain, M.; Lapin, Z.; Sáenz, J.J.; Wicks, G.; Novotny, L. Demonstration of Zero Optical Backscattering from Single Nanoparticles. Nano Lett. 2013, 13, 1806-1809. [CrossRef] [PubMed]

15. Ioffe, Z.; Shamai, T.; Ophir, A.; Noy, G.; Yutsis, I.; Kfir, K.; Cheshnovsky, O.; Selzer, Y. Detection of Heating in Current-Carrying Molecular Junctions by Raman Scattering. Nat. Nanotechnol. 2008, 3, 727-732. [CrossRef] [PubMed]

16. Ward, D.R.; Corley, D.A.; Tour, J.M.; Natelson, D. Vibrational and Electronic Heating in Nanoscale Junctions. Nat. Nanotechnol. 2011, 6, 33-38. [CrossRef] [PubMed]

17. Torres-Torres, C. Ablation and Optical Third-Order Nonlinearities in Ag Nanoparticles. Int. J. Nanomed. 2010, 5, 925. [CrossRef] [PubMed]

18. Plech, A.; Kotaidis, V.; Grésillon, S.; Dahmen, C.; Von Plessen, G. Laser-Induced Heating and Melting of Gold Nanoparticles Studied by Time-Resolved x-Ray Scattering. Phys. Rev. B 2004, 70, 195423. [CrossRef]

19. King, M.D.; Khadka, S.; Craig, G.A.; Mason, M.D. Effect of Local Heating on the SERS Efficiency of Optically Trapped Prismatic Nanoparticles. J. Phys. Chem. C 2008, 112, 11751-11757. [CrossRef]

20. Gutiérrez, Y.; Alcaraz, R.; Osa, D.; Ortiz, D.; Saiz, J.M.; González, F.; Moreno, F. Plasmonics in the Ultraviolet with Aluminum, Gallium, Magnesium and Rhodium. Appl. Sci. 2018, 8, 64. [CrossRef]

21. Sharma, B.; Frontiera, R.R.; Henry, A.-I.; Ringe, E.; Van Duyne, R.P. SERS: Materials, Applications, and the Future. Mater. Today 2012, 15, 16-25. [CrossRef]

22. Yang, Y.; Callahan, J.M.; Kim, T.H.; Brown, A.S.; Everitt, H.O. Ultraviolet Nanoplasmonics: A Demonstration of Surface-Enhanced Raman Spectroscopy, Fluorescence, and Photodegradation Using Gallium Nanoparticles. Nano Lett. 2013, 13, 2837-2841. [CrossRef] [PubMed]

23. Taguchi, A. Plasmonic Tip for Nano Raman Microcopy: Structures, Materials, and Enhancement. Opt. Rev. 2017, 24, 462-469. [CrossRef]

24. Honda, M.; Kumamoto, Y.; Taguchi, A.; Saito, Y.; Kawata, S. Efficient UV Photocatalysis Assisted by Densely Distributed Aluminum Nanoparticles. J. Phys. D Appl. Phys. 2015, 48, 184006. [CrossRef]

25. Watson, A.M.; Zhang, X.; Alcaraz de la Osa, R.; Marcos Sanz, J.; González, F.; Moreno, F.; Finkelstein, G.; Liu, J.; Everitt, H.O. Rhodium Nanoparticles for Ultraviolet Plasmonics. Nano Lett. 2015, 15, 1095-1100. [CrossRef] [PubMed]

26. Zhang, X.; Li, X.; Zhang, D.; Su, N.Q.; Yang, W.; Everitt, H.O.; Liu, J. Product Selectivity in Plasmonic Photocatalysis for Carbon Dioxide Hydrogenation. Nat. Commun. 2017, 8, 14542. [CrossRef] [PubMed]

27. Shuang, S.; Lv, R.; Xie, Z.; Zhang, Z. Surface Plasmon Enhanced Photocatalysis of Au/Pt-Decorated TiO2 Nanopillar Arrays. Sci. Rep. 2016, 6, 26670. [CrossRef] [PubMed]

28. Seh, Z.W.; Liu, S.; Zhang, S.-Y.; Shah, K.W.; Han, M.-Y. Synthesis and Multiple Reuse of Eccentric Au@TiO2 Nanostructures as Catalysts. Chem. Commun. 2011, 47, 6689-6691. [CrossRef] [PubMed]

29. Linic, S.; Christopher, P.; Ingram, D.B. Plasmonic-Metal Nanostructures for Efficient Conversion of Solar to Chemical Energy. Nat. Mater. 2011, 10, 911-921. [CrossRef] [PubMed]

30. Sanz, J.M.; Ortiz, D.; Alcaraz de la Osa, R.; Saiz, J.M.; González, F.; Brown, A.S.; Losurdo, M.; Everitt, H.O.; Moreno, F. UV Plasmonic Behavior of Various Metal Nanoparticles in the Near- and Far-Field Regimes: Geometry and Substrate Effects. J. Phys. Chem. C 2013, 117, 19606-19615. [CrossRef]

31. Hou, W.; Cronin, S.B. A Review of Surface Plasmon Resonance-Enhanced Photocatalysis. Adv. Funct. Mater. 2013, 23, 1612-1619. [CrossRef]

32. Montini, T.; Melchionna, M.; Monai, M.; Fornasiero, P. Fundamentals and Catalytic Applications of CeO2 -Based Materials. Chem. Rev. 2016, 116, 5987-6041. [CrossRef] [PubMed]

33. Held, G. Introduction to Light Emitting Diode Technology and Applications; Auerbach Publications: New York, NY, USA, 2008.

34. Taniyasu, Y.; Kasu, M.; Makimoto, T. An Aluminium Nitride Light-Emitting Diode with a Wavelength of 210 Nanometres. Nature 2006, 441, 325-328. [CrossRef] [PubMed] 
35. Mochalin, V.N.; Shenderova, O.; Ho, D.; Gogotsi, Y. The Properties and Applications of Nanodiamonds. Nat. Nanotechnol. 2012, 7, 11-23. [CrossRef] [PubMed]

36. Absorption and Scattering of Light by Small Particles; Bohren, C.F.; Huffman, D.R. (Eds.) Wiley-VCH Verlag GmbH: Weinheim, Germany, 1998.

37. Mie, G. Beiträge Zur Optik Trüber Medien, Speziell Kolloidaler Metallösungen. Ann. Phys. 1908, 330, 377-445. [CrossRef]

38. Shibanuma, T.; Albella, P.; Maier, S.A. Unidirectional Light Scattering with High Efficiency at Optical Frequencies Based on Low-Loss Dielectric Nanoantennas. Nanoscale 2016, 8, 14184-14192. [CrossRef] [PubMed]

39. COMSOL Multiphysics v5.2; COMSOL AB: Stockholm, Sweden, 2016; Available online: www.comsol.com (accessed on 22 October 2018).

40. Govorov, A.O.; Richardson, H.H. Generating Heat with Metal Nanoparticles We Describe Recent Studies on Photothermal Effects Using Colloidal. Rev. Lit. Arts Am. 2007, 2, 30-38.

41. Baffou, G.; Quidant, R. Thermo-Plasmonics: Using Metallic Nanostructures as Nano-Sources of Heat. Laser Photonics Rev. 2013, 7, 171-187. [CrossRef]

42. Wang, Q.; Cao, W.; Kuang, J.; Jiang, P. Spherical AlN Particles Synthesized by the Carbothermal Method: Effects of Reaction Parameters and Growth Mechanism. Ceram. Int. 2018, 44, 4829-4834. [CrossRef]

43. Chen, X.; Mao, S.S. Titanium Dioxide Nanomaterials: Synthesis, Properties, Modifications, and Applications. Chem. Rev. 2007, 107, 2891-2959. [CrossRef] [PubMed]

44. Tamizhdurai, P.; Sakthinathan, S.; Chen, S.-M.; Shanthi, K.; Sivasanker, S.; Sangeetha, P. Environmentally Friendly Synthesis of CeO2 Nanoparticles for the Catalytic Oxidation of Benzyl Alcohol to Benzaldehyde and Selective Detection of Nitrite. Sci. Rep. 2017, 7, 46372. [CrossRef] [PubMed]

45. Hwang, S.Y.; Kim, T.J.; Jung, Y.W.; Barange, N.S.; Park, H.G.; Kim, J.Y.; Kang, Y.R.; Kim, Y.D.; Shin, S.H.; Song, J.D.; et al. Dielectric Function and Critical Points of AlP Determined by Spectroscopic Ellipsometry. J. Alloys Compd. 2014, 587, 361-364. [CrossRef]

46. Garriga, M.; Lautenschlager, P.; Cardona, M.; Ploog, K. Optical Properties of AlAs. Solid State Commun. 1987, 61, 157-160. [CrossRef]

47. Khoshman, J.M.; Kordesch, M.E. Optical Characterization of Sputtered Amorphous Aluminum Nitride Thin Films by Spectroscopic Ellipsometry. J. Non. Cryst. Solids 2005, 351, 3334-3340. [CrossRef]

48. Shih, W.C.; Chen, C.H.; Chiu, F.C.; Lai, C.M.; Hwang, H.L. CeO2 Optical Properties and Electrical Characteristics. ECS Trans. 2010, 28, 435-442.

49. Devore, J.R. Refractive Indices of Rutile and Sphalerite. J. Opt. Soc. Am. 1951, 41, 416-419. [CrossRef]

50. Palik, E.D. Handbook of Optical Constants of Solids; Academic Press: New York, NY, USA, 1998.

51. Rakovich, A.; Albella, P.; Maier, S.A. Plasmonic Control of Radiative Properties of Semiconductor Quantum Dots Coupled to Plasmonic Ring Cavities. ACS Nano 2015, 9, 2648-2658. [CrossRef] [PubMed]

52. Knight, M.W.; King, N.S.; Liu, L.; Everitt, H.O.; Nordlander, P.; Halas, N.J. Aluminum for Plasmonics. ACS Nano 2014, 8, 834-840. [CrossRef] [PubMed]

53. Gutierrez, Y.; Ortiz, D.; Sanz, J.M.; Saiz, J.M.; Gonzalez, F.; Everitt, H.O.; Moreno, F. How an Oxide Shell Affects the Ultraviolet Plasmonic Behavior of Ga, Mg, and Al Nanostructures. Opt. Express 2016, 24, 20621. [CrossRef] [PubMed]

54. Barreda, Á.I.; Gutiérrez, Y.; Sanz, J.M.; González, F.; Moreno, F. Light Guiding and Switching Using Eccentric Core-Shell Geometries. Sci. Rep. 2017, 7, 11189. [CrossRef] [PubMed]

55. Albella, P.; Shibanuma, T.; Maier, S.A. Switchable Directional Scattering of Electromagnetic Radiation with Subwavelength Asymmetric Silicon Dimers. Sci. Rep. 2015, 5, 18322. [CrossRef] [PubMed]

56. Maksymov, I.S.; Staude, I.; Miroshnichenko, A.E.; Kivshar, Y.S. Optical Yagi-Uda Nanoantennas. Nanophotonics 2012, 1, 65-81. [CrossRef]

57. Shibanuma, T.; Maier, S.A.; Albella, P. Polarization Control of High Transmission/Reflection Switching by All-Dielectric Metasurfaces. Appl. Phys. Lett. 2018, 112, 063103. [CrossRef]

58. Rechberger, W.; Hohenau, A.; Leitner, A.; Krenn, J.R.; Lamprecht, B.; Aussenegg, F.R. Optical Properties of Two Interacting Gold Nanoparticles. Opt. Commun. 2003, 220, 137-141. [CrossRef]

(C) 2018 by the authors. Licensee MDPI, Basel, Switzerland. This article is an open access article distributed under the terms and conditions of the Creative Commons Attribution (CC BY) license (http://creativecommons.org/licenses/by/4.0/). 